$$
\begin{aligned}
& \text { BNL - 71791-2003-CP } \\
& \text { CAP-382-Beam-03C }
\end{aligned}
$$

\title{
BNL Very Long Baseline Experiment with a Super Neutrino Beam
}

\author{
Stephen Kahn \\ Brookhaven National Laboratory
}

October 2003

\section{CENTER FOR ACCELERATOR PHYSICS}

\author{
BROOKHAVEN NATIONAL LABORATORY \\ BROOKHAVEN SCIENCE ASSOCIATES \\ Under Contract No. DE-AC02-98CH10886 with the \\ UNITED STATES DEPARTMENT OF ENERGY
}

Presented at "NuFact03 Workshop", Columbia University, New York City, June 5-11, 2003 


\section{DISCLAIMER}

This report was prepared as an account of work sponsored by an agency of the United States Government. Neither the United States Government nor any agency thereof, nor any of their employees, nor any of their contractors, subcontractors, or their employees, makes any warranty, express or implied, or assumes any legal liability or responsibility for the accuracy, completeness, or usefulness of any information, apparatus, product, or process disclosed, or represents that its use would not infringe privately owned rights. Reference herein to any specific commercial product, process, or service by trade name, trademark, manufacturer, or otherwise, does not necessarily constitute or imply its endorsement, recommendation, or favoring by the United States Government or any agency, contractor or subcontractor thereof. The views and -opinions of authors expressed herein do not necessarily state or reflect those of the United States Government or any agency, contractor or subcontractor thereof. 


\title{
BNL Very Long Baseline Experiment With a Super Neutrino Beam
}

\author{
Stephen Kahn \\ Brookhaven National Laboratory, Upton, NY, 11973
}

\begin{abstract}
An upgrade to the BNL AGS could produce a very intense proton source at a relatively low cost. This proton source could produce a conventional neutrino beam with a very significant flux at large distances from the laboratory. In this paper we examine the possibility of using this neutrino beam for a very long baseline oscillation experiment where a 500 kiloton water Cherenkov detector is situated at the Homestake mine in South Dakota. We study the physics potential of a high intensity neutrino oscillation experiment with a $2540 \mathrm{~km}$ baseline and a peak neutrino energy of $-1 \mathrm{GeV}$.
\end{abstract}

\section{INTRODUCTION}

Recent results have shown that neutrinos can oscillate between flavor states. This has created interest in understanding the fundamental aspects of neutrino oscillations. To explore the physics of the neutrino masses and mixing angles will require new facilities with intense proton beam sources. A working group at Brookhaven National Laboratory is studying the feasibility of upgrading the AGS to a $1.0 \mathrm{MW}$ proton sources and using it to create an intense neutrino super-beam capable of producing a significant flux at a large distance from BNL. The AGS upgrade would increase the repetition rate of the accelerator from 0.5 to $2.5 \mathrm{~Hz}$ with $8.9 \times 10^{13}$ protons per pulse. The expected integrated intensity for a typical year of operation $\left(10^{7} \mathrm{sec}\right.$. $)$ is expected to be $2.2 \times 10^{21} 28 \mathrm{GeV}$ protons on target. A description of the accelerator, target and horn system, and $v$ beamline of this super-beam facility is given in a report by the working group [1]. The physics potential of this proposed long baseline experiment is described in another report $[2,3]$. The goal of this facility is to have the ability to measure all of the parameters of the SMN matrix in a single experiment. This includes:

- A precise determination of the oscillation disappearance parameters $\Delta \mathrm{m}_{32}{ }^{2}$ and $\sin ^{2} 2 \theta_{23}$.

- The detection of the appearance oscillation $\nu_{\mu} \rightarrow v_{e}$ and the measurement of $\sin ^{2} 2 \theta_{13}$.

- The measurement of $\Delta \mathrm{m}^{2}{ }_{21} \sin ^{2} 2 \theta_{12}$ in a $v_{\mu} \rightarrow v_{\mathrm{e}}$ appearance mode.

- The verification of the matter enhancement and the sign of $\Delta \mathrm{m}_{32}{ }^{2}$.

- The determination of the $\mathrm{CP}$-violation parameter $\delta_{\mathrm{CP}}$ in the neutrino sector.

The useful neutrino energy range is restricted at $\sim 1 \mathrm{GeV}$ on the low end by Fermi motion in the nucleus and by $\sim 6 \mathrm{GeV}$ on the high end by background from inelastic $v$ 
interactions. Placing the detector at the Homestake Mine, which is 2540 kilometers from BNL, allows the oscillation phase to vary from below $\pi / 2$ to above $5 \pi / 2$ for $\Delta \mathrm{m}_{32}{ }^{2}=0.0025 \mathrm{eV}^{2}$, which would clearly demonstrate oscillations in the energy distribution and provide a precise measurement of $\Delta \mathrm{m}_{32}{ }^{2}$. For the purposes of calculating expected event rates and backgrounds a 0.5 megaton water Cherenkov detector is used. A neutrino beam directed at the Homestake Mine must be inclined into the ground by $11.3^{\circ}$ with respect to the surface. This incline restricts the location of the close-in detector to be just outside the beam dump at $275 \mathrm{~m}$ from the target.

\section{FLUX CALCULATIONS AND EVENT ESTIMATES}

Neutrino flux spectra have been calculated for the upgraded AGS with a horn design proposed for this beam [4]. Fig 1a shows the expected $\nu_{\mu}$ and $v_{\mathrm{e}}$ flux distributions for this beam in units of $v / \mathrm{GeV} / \mathrm{m}^{2} /$ Proton-on-target at $1 \mathrm{~km}$ from the target. The expected $v_{\mathrm{e}}$ event contamination is $\sim 1 \%$ in this wide-band beam. At distances greater than $1 \mathrm{~km}$ the spectrum is not strongly dependent on the on the $v$ source position and the consequently the flux at the detector scales with $r^{-2}$. The table in Fig $1 \mathrm{~b}$ shows the number of events expected at $2540 \mathrm{~km}$ in a 0.5 megaton water Cherenkov detector for a $5 \times 10^{7}$ sec running period. We expect to see 52000 charged current and 17000 neutral current events during that run. The event analysis in a water Cherenkov detector will concentrate on the quasi-elastic samples since the neutrino energy is better known and the backgrounds in the $v_{\mathrm{e}}$ appearance are better understood. A liquid argon detector would be a better detector to analyze the multiple particle channels.

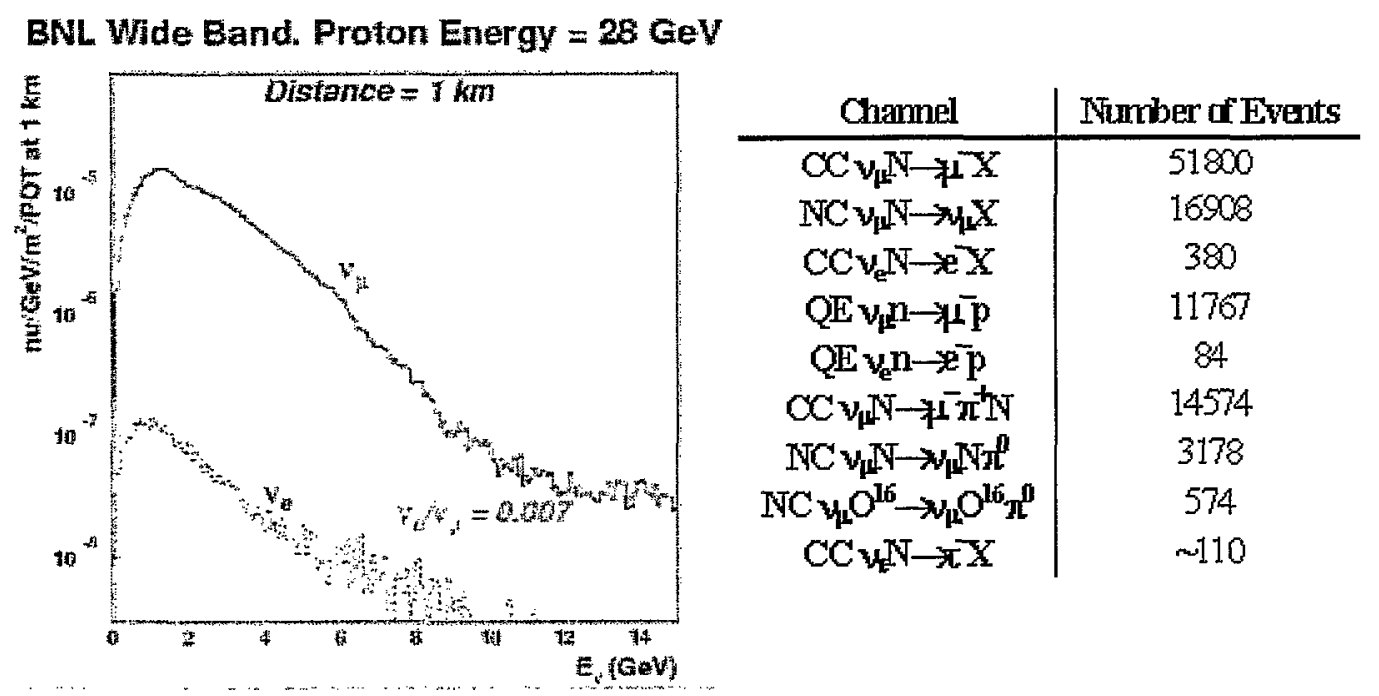

Figure 1a: The $v_{\mu}$ and $v_{\mathrm{e}}$ flux spectra from $28 \mathrm{GeV}$ protons on a graphite target seen at $1 \mathrm{~km}$. b: Table of events seen in neutrino induced channels at $2540 \mathrm{~km}$ during a $5 \times 10^{7} \mathrm{sec}$ run in a 0.5 megaton water Cherenkov Detector. 


\section{$v_{\mu}$ DISAPPEARNCE}

Figure 2a shows the energy spectrum of $v_{\mu}$ quasi-elastic channel for a running period of $5 \times 10^{7}$ seconds with a $1 \mathrm{MW}$ beam and a 0.5 megaton water Cherenkov detector at $2540 \mathrm{~km}$. The top curve shows the event spectrum without oscillations. The middle curve with error bars shows the event spectrum with oscillations when $\Delta \mathrm{m}_{32}{ }^{2}=0.0025 \mathrm{eV}^{2}$ and $\sin ^{2} 2 \theta_{23}=1$. In this figure we have assumed that the resolution in the reconstructed $v$ energy $\left(\mathrm{E}_{v}\right)$ is $10 \%$, which should be achievable with $10 \%$ photo multiplier tube coverage. This does not include a systematic error from the calibration of the overall detector energy scale. A great advantage of the very long baseline and the multiple oscillation pattern is that the systematic errors for flux normalization, background subtraction, and nuclear effects are small. The background shown in the lower curve of the figure comes from non quasi-elastic charged current events that also oscillate and will tend to smear the nodal pattern.
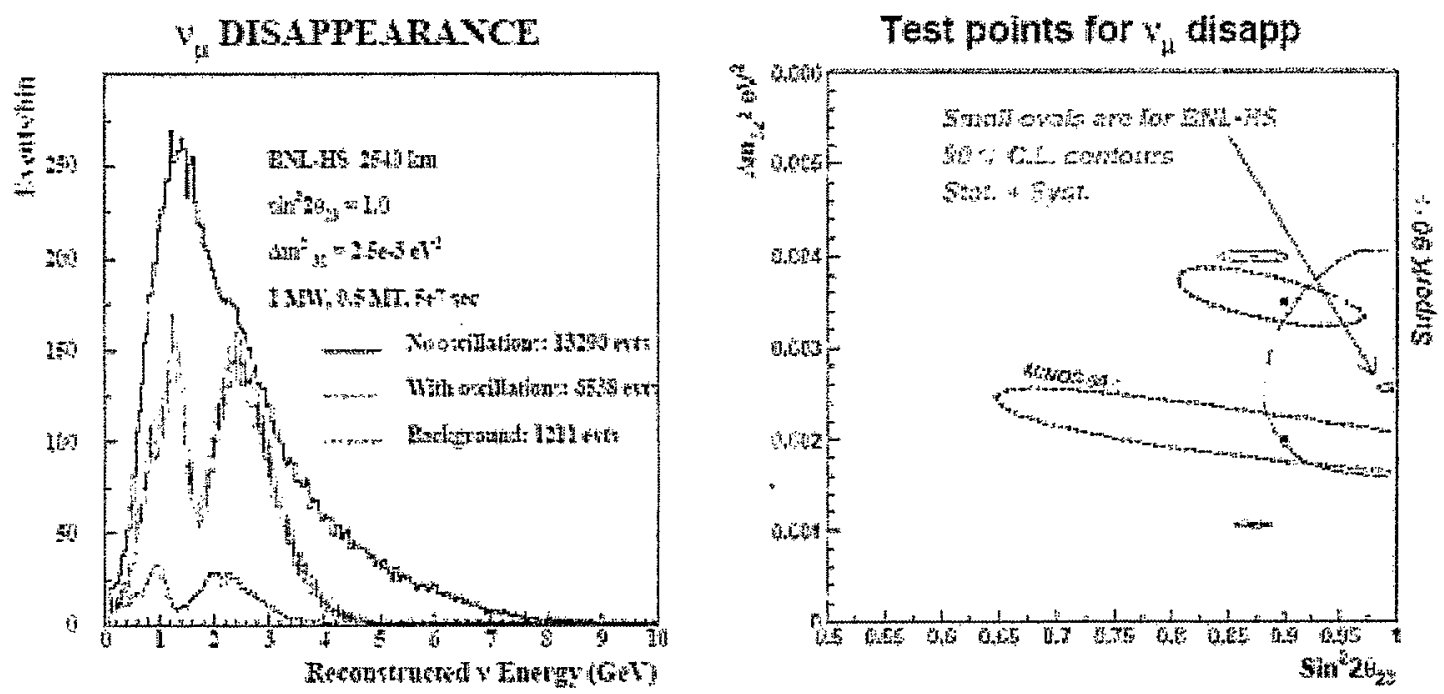

Figure 2a: $v_{\mu}$ Quasi-elastic event spectrum. Curves show events without oscillations, with oscillations, and the background to oscillated $\mathrm{QE}$ events. The oscillated events assume that $\Delta \mathrm{m}_{32}{ }^{2}=0.0025 \mathrm{eV}^{2}$. b: A comparison of the precision of the measurement of $\Delta \mathrm{m}_{32}{ }^{2}$ and $\sin ^{2} 2 \theta_{23}$ for this experiment and that expected by the MNOS experiment and seen by the Super-Kamiokande experiment.

Figure $2 \mathrm{~b}$ shows a comparison of the experimental precision of $\Delta \mathrm{m}_{32}{ }^{2}$ and $\sin ^{2} 2 \theta_{23}$ expected in this experiment with those seen in the Super-Kamiokande experiment and expected in the MINOS experiment.

\section{$v_{\mu} \rightarrow v_{\mathrm{e}}$ APPEARANCE}

The measurement of $\theta_{13}, \delta_{\mathrm{CP}}$ and $\Delta \mathrm{m}_{21}{ }^{2}$ and the sign of $\Delta \mathrm{m}_{32}{ }^{2}$ can be extracted from the wideband $v_{e}$ appearance spectrum. The probability for the appearance of $v_{\mu} \rightarrow v_{e}$ oscillations from 3-generation mixing including matter effects can be expressed analytically [3]. Figure $3 a$ shows the expected $v_{e}$ event distribution for $\sin ^{2} 2 \theta_{13}=0.04$ 
and $\Delta \mathrm{m}_{32}{ }^{2}=0.0025 \mathrm{eV}^{2}$ and the same running conditions previously mentioned. The matter enhancement (suppression) for the normal (reversed) $\Delta \mathrm{m}_{32}{ }^{2}$ sign ordering dominates the spectrum for $E_{v}>3 \mathrm{GeV}$. Sensitivity to the $C P$ phase is greatest for $E_{v}$ range from 1 to $3 \mathrm{GeV}$. The effect of the solar oscillation from $\Delta \mathrm{m}_{21}{ }^{2}$ is largest at low $\mathrm{E}_{v}$. Figure $3 \mathrm{~b}$ shows the event spectrum with $\sin ^{2} 2 \theta_{13}=0$. The appearance mode is more sensitive to the presence of backgrounds. The main sources of background come from $v_{\mathrm{e}}$ contamination in the beam and neutral current reactions that have a single $\pi^{\circ}$ that are misidentified as electrons. An estimate of these backgrounds is shown in figure 3. There is concern at this point that the detection efficiency of $\pi^{\circ}$ is overestimate in the current simulation. This is being investigated.
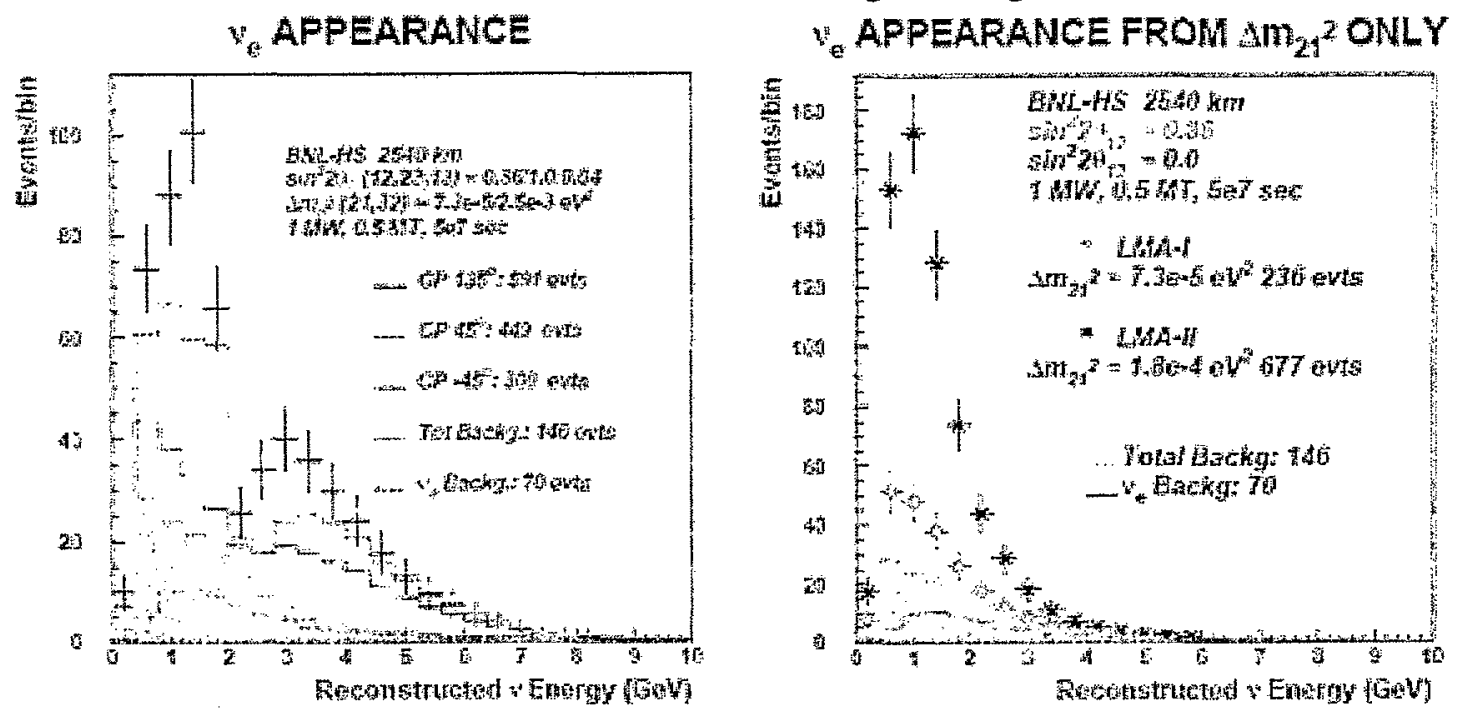

Figure 3a: The expected $v_{e}$ event spectrum for $\sin ^{2} 2 \theta_{13}=0.04$ and for 3 values of $\delta_{\text {CP. }}$ The figure also shows the total background for $v_{e}$. b: The expected spectrum for $\sin ^{2} 2 \theta_{13}=0$. The resultant spectrum shows $\Delta \mathrm{m}_{21}^{2}$ oscillations.

\section{ACKNOWLEDGMENTS}

This work was performed with the support of the US DOE under Contract No. DEAC02-98CH10886.

\section{REFERENCES}

1. The AGS-Based Super Neutrino Beam Facility, The BNL Neutrino Working Group Report II, BNL-71228-2003-IR.

2. Report of the BNL Neutrino Working Group: Very Long Baseline Neutrino Oscillation Experiment for Precise Determination of Oscillation Parameters and Search for $\nu_{\mu} \rightarrow \nu_{\varepsilon}$ Appearance and CP Violation, BNL Report 69395 (2002).

2. M.V. Diwan et al., Physical Review D 68, 012002 (2002)

4. S.A. Kahn et al., Focusing Horn System for the BNL...,Proc of the 2003 Particle Accelerator Conference, BNL-71411-2003-CP. 